\title{
Molecular Understanding of Energy Storage in Supercapacitors with Porous Graphyne Electrodes: From Semiconductor to Conductor
}

\section{Tangming Mo}

Huazhong University of Science and Technology

\section{Zhenxiang Wang}

Huazhong University of Science and Technology

\section{Liang Zeng}

Huazhong University of Science and Technology

\section{Ming Chen}

State Key Laboratory of Coal Combustion, School of Energy and Power Engineering, Huazhong University of Science and Technology (HUST) https://orcid.org/0000-0002-2188-5472

\section{Yong-Qing Zhao}

\author{
Lanzhou University
}

\section{Guang Feng ( $\nabla$ gfeng@hust.edu.cn )}

Huazhong University of Science and Technology https://orcid.org/0000-0001-6659-9181

\section{Article}

Keywords: Two-dimensional Porous Materials, Charge Storage, Charging Dynamics, Double-layer Capacitances, Quantum Capacitances

Posted Date: July 12th, 2021

DOl: https://doi.org/10.21203/rs.3.rs-640397/v1

License: (c) (1) This work is licensed under a Creative Commons Attribution 4.0 International License. Read Full License 

Porous Graphyne Electrodes: From Semiconductor to Conductor

3 Tangming Mo, ${ }^{1,2}$ Zhenxiang Wang, ${ }^{1,2}$ Liang Zeng, ${ }^{1,2}$ Ming Chen, ${ }^{1,2}$ Yongqing Zhao, ${ }^{3}$

4 Guang Feng ${ }^{1,2, *}$

5 Two-dimensional (2D) porous materials with high specific surface area and ordered morphology exhibit great potential as supercapacitor electrodes. The fundamental understanding of the charge storage and charging dynamics of 2D porous materials can help the optimal design of supercapacitors. Herein, we investigated the energy storage, including the double layer and quantum capacitances, of supercapacitors with typical 2D porous graphynes in the ionic liquid electrolyte by combining molecular dynamics simulation and density functional theory. Simulations revealed that supercapacitors with porous graphyne electrodes could obtain excellent double-layer capacitances, but their total capacitances are limited by the low quantum capacitances. We further predicted boron/nitrogen-doped graphynes and found that the new porous graphynes turn into good conductors after doping and could achieve a quite high quantum capacitance. The charging dynamics in nanoscale and capacitive performance in macroscale based on the predicted graphyne electrodes were evaluated by combining molecular simulation and transmission line model. Results demonstrate that both outstanding gravimetric and volumetric energy and power densities could be obtained in doped porous graphyne supercapacitors. These findings pave the way for understanding energy storage mechanisms and designing highperformance supercapacitors.

\footnotetext{
${ }^{1}$ State Key Laboratory of Coal Combustion, School of Energy and Power Engineering, Huazhong University of Science and Technology (HUST), Wuhan 430074, China. ${ }^{2}$ Nano Interface Centre for Energy, School of Energy and Power Engineering, Huazhong University of Science and Technology, 430074, China. ${ }^{3}$ State Key Laboratory of Applied Organic Chemistry, Laboratory of Special Function Materials and Structure Design of the Ministry of Education, College of Chemistry and Chemical Engineering, Lanzhou University, Lanzhou 730000, China. T.M.M. and Z.X.W. contributed equally to this work. Corresponding author's email: gfeng@hust.edu.cn
} 
Supercapacitors can provide fast charging and high power density but moderate energy density compared to batteries ${ }^{1,2}$. Enhancing their energy density without compromising the power density can make their more widespread applications. Porous carbons have been widely used as electrode materials in supercapacitors to enhance their energy storage ${ }^{3-5}$. The amorphous porous carbons (e.g., activated carbons ${ }^{6}$ and carbide-derived carbons ${ }^{7}$ ) are the most popular due to their large specific surface area and high electronic conductivity ${ }^{5}$. However, the random morphology structure would lead to long ion motion paths and severe ion collisions, thereby slowing down the charging dynamics and reducing power density $5,8,9$.

Conductive 2D porous materials, with regular porosities and superior chemical stability, have attracted growing research interest as electrodes for supercapacitors ${ }^{10-14}$. Sheberla et al. revealed that the supercapacitor with electrodes made entirely of the conductive metal-organic framework (MOF) could obtain high areal capacitance and low cell resistance in an organic electrolyte $^{10}$. Supercapacitors with hexaaminobenzene-derived MOF electrodes and aqueous electrolytes were measured to have both high volumetric and high areal capacitances ${ }^{12}$. Combining molecular dynamics (MD) simulations and experimental measurements, conductive MOFs in an ionic liquid (IL) were uncovered to achieve unprecedentedly high volumetric energy and power densities ${ }^{13}$. Nevertheless, those conductive MOFs only have a moderate specific surface area, and therefore, more 2D porous electrodes need to be developed. Porous graphynes, such as hydrogen substituted graphyne (HsGY) ${ }^{15}$, hydrogen substituted graphdiyne (HsGDY) with $\mathrm{AA}$ stacking ${ }^{16}$ and $\mathrm{AB}$ stacking ${ }^{17}$ structures, and fluoride substituted graphdiyne ${ }^{18}$, with extended $\pi$-conjugated carbon skeleton of porous structure and high specific surface area are synthesized and have been applied to lithium and sodium ion storage ${ }^{17-19}$ and supercapacitor ${ }^{20}$. For example, supercapacitors based on HsGDY electrodes and $6 \mathrm{M} \mathrm{KOH}$ aqueous electrolyte could achieve a high specific capacitance $(230 \mathrm{~F} / \mathrm{g})^{20}$.

However, the band gaps have been found in both $\mathrm{HsGY}^{15}$ and HsGDY' ${ }^{21}$, implying that they are not good conductors. Previous studies showed that low-dimensional materials with low density of states (DOS) near Fermi level, such as graphene ${ }^{22,23}$ and carbon nanotube ${ }^{24,25}$, have a non-negligible quantum capacitance because it will reduce total capacitance significantly. Nevertheless, existent research on quantum capacitance is limited to open surface electrodes. It is still unclear how quantum capacitance will influence the performance of supercapacitors based 
on the porous electrode.

In this work, we performed constant-potential MD simulations to investigate the electrical double layer (EDL) capacitances of HsGDY-based supercapacitors with two stacking structures (i.e., $\mathrm{AA}$ and $\mathrm{AB}$ stacking) and two porous graphynes with different pore size (i.e., HsGY and hydrogen substituted graphtriyne, HsGTY), in IL 1-ethyl-3-methylimidazolium tetrafluoroborate [EMIM] $\left[\mathrm{BF}_{4}\right]$ (Fig. S1 and details see Methods and Supplementary part 1). Density functional theory (DFT) was employed to calculate their quantum capacitances (details in Methods). Results reveal that those porous electrodes could obtain high EDL performance but are limited by their low quantum capacitances. The new structures of graphyne-based electrodes are further predicted with doping and found to be well conductive and possess excellent quantum capacitance. The doped graphyne supercapacitors exhibit outstanding energy and power densities by combining MD simulation and transmission line model (TLM).

\section{Results}

\section{In-pore double-layer structure}

We begin our analysis by examining the microstructure of electrolytes inside pores. Figure 1a displays the in-plane charge and ion distribution across EDLs formed by ILs and AA stacking HsGDY under different polarizations. At the potential of zero charge (PZC), cations and anions mix, forming a hexagonal pattern in the plane close to the pore. After polarization, cations and anions become more heterogenous: counterions (cations at negative electrode and anions at positive electrode) are adsorbed closer to the pore surface, while co-ions are repulsed from the pore surface to the center. The radial distribution exhibits more detailed layering, as shown in Fig. 1b. Co-ions form a two-layer structure inside the pore under weaker polarization: one layer near the pore surface and the other near the center (Fig. 1b and Fig. S2a), but this distribution will transit to a one-layer structure as the polarization gets stronger. As for axial distribution shown in Fig. 1c, both cations and anions settle in the interlayer space of HsGDY at the PZC and under positive polarization. But under negative polarization, alternated distribution of ions is found: cations accumulate in the interlayer space, and anions accumulate in the HsGDY sheets. Moreover, cations distribute more homogenously in axial direction under negative polarization than at PZC or under positive polarization due to their more random orientation (Fig. S2c). 
a
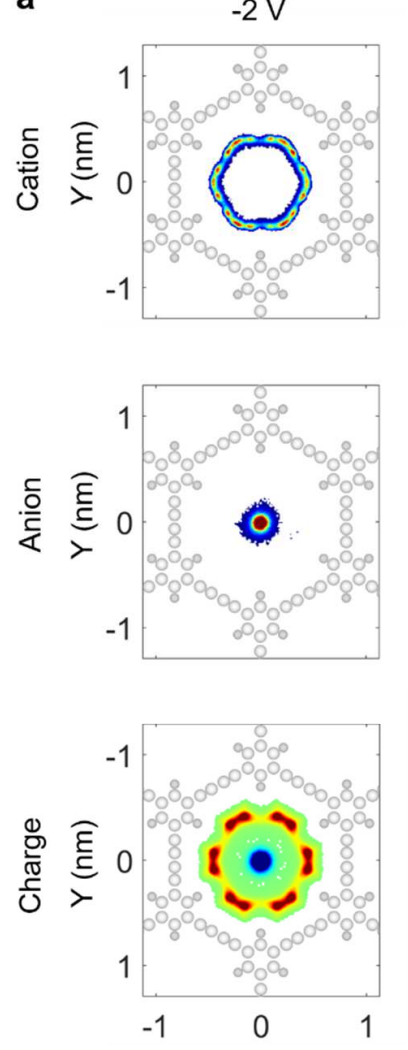

$X(\mathrm{~nm})$
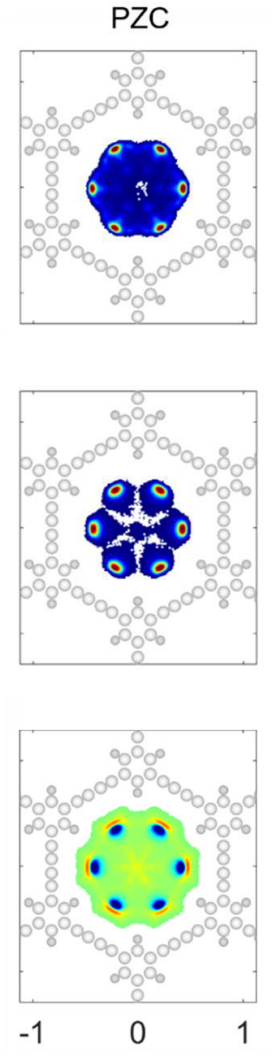

$\mathrm{X}(\mathrm{nm})$
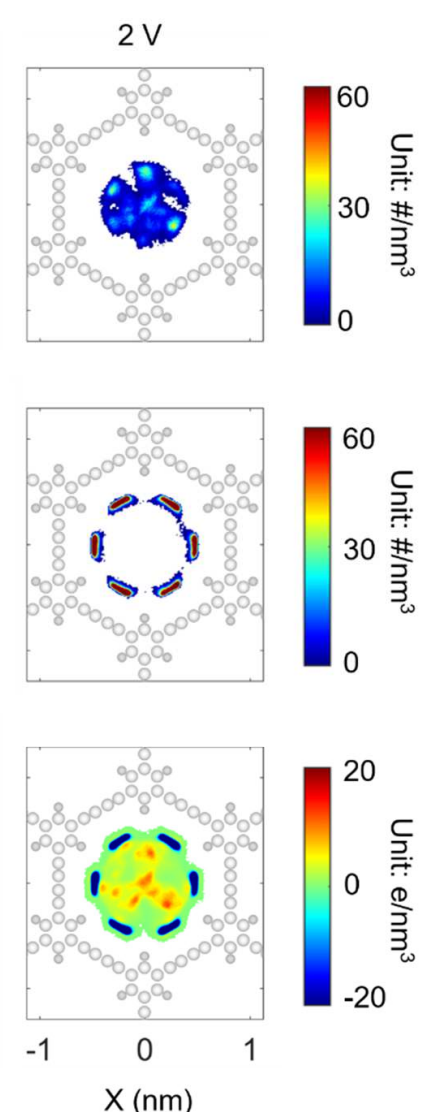

b

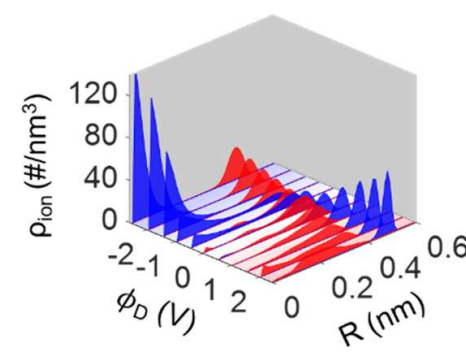

C
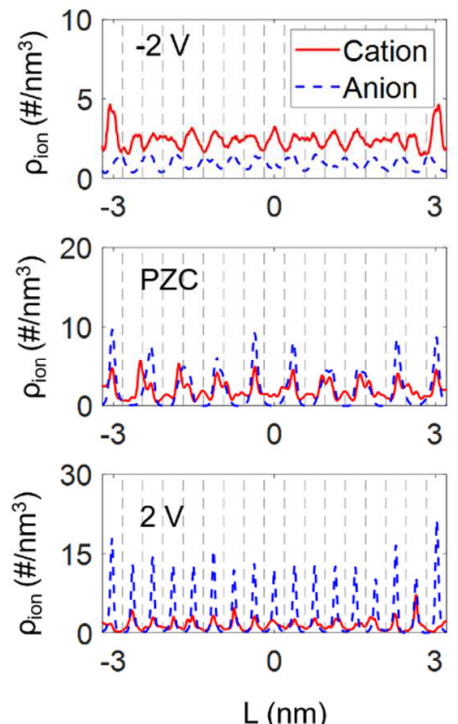

Fig. 1. EDL structure inside AA stacking HsGDY. a, In-plane maps of ion and charge distributions. Upper, middle, and bottom rows refer to negative polarization $(-2 \mathrm{~V}), \mathrm{PZC}$, and positive polarization (2 V), respectively. In each plane, spheres represent the atoms of HsGDY (the white and gray ones are

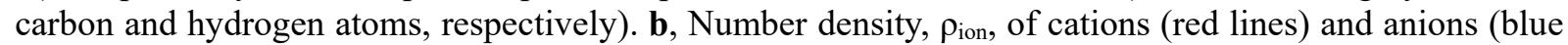
lines) along with the radial distributions, $R$, at different electrode potentials, $\phi_{D}$. c, Number density of ion,

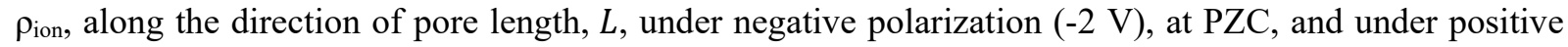
polarization $(2 \mathrm{~V})$. The dotted lines refer to the positions of HsGDY layers.

For AB stacking HsGDY, the pore is divided into two parts (Fig. S3a): the larger part can be wetted by the ions at the PZC and thus defined as a channel; while the smaller part is named as a cage because it shows ionophobicity and only counterions could be adsorbed into this part under polarization. Interestingly, the in-plane ion distributions in the smaller pore (HsGY, Fig. S4) and larger pore (HsGTY, Fig. S5) exhibit a petal pattern and a round pattern, respectively. The distributions of cations and anions in $\mathrm{HsGY}$ are homogeneous and almost immune to polarization (Fig. S4c); while in HsGTY, more layers of ions along the radial direction are observed, especially under negative polarization, and the orientation of cation is distributed more randomly (Fig. S5b vs. Fig. S2b and Fig. S4b). 
a

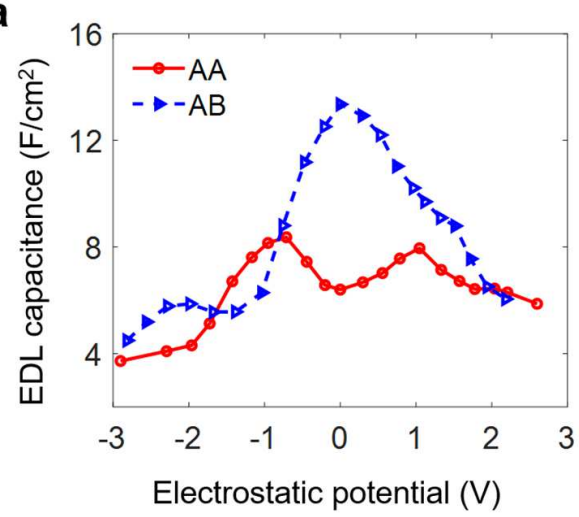

C

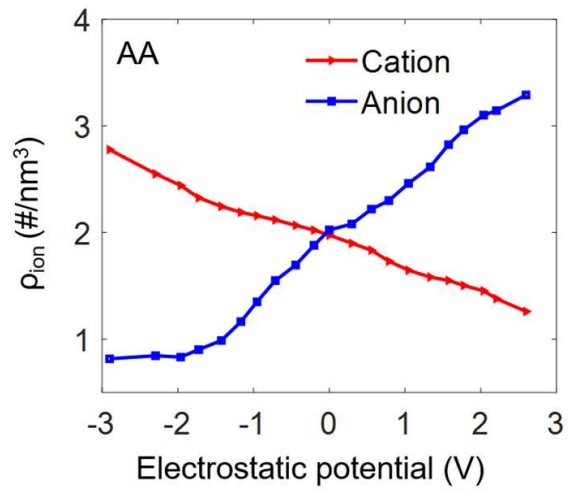

e

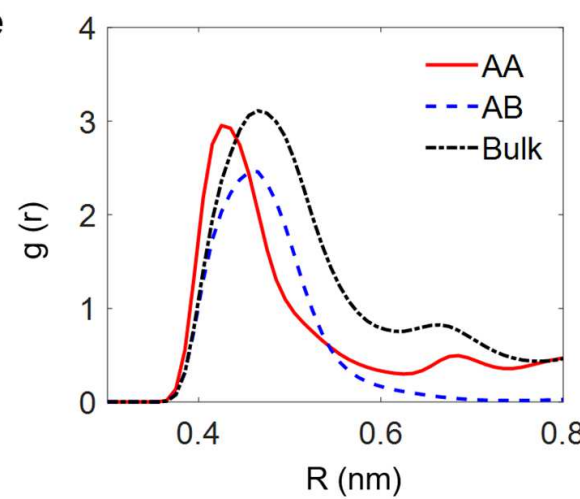

b

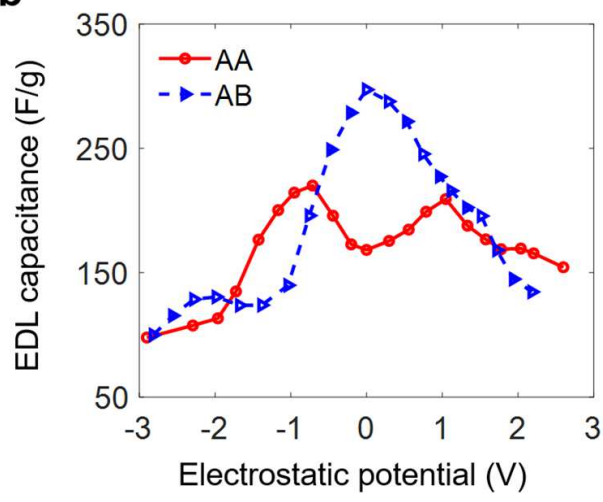

d

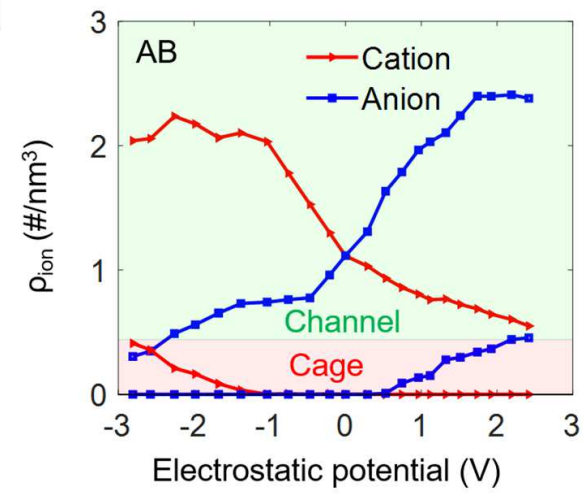

f

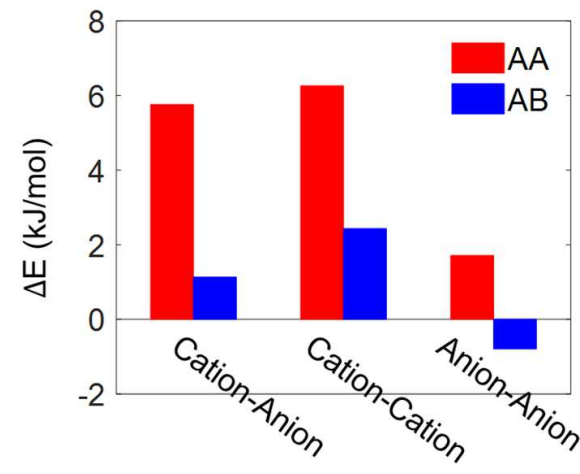

Fig. 2. Electrical double-layer capacitances and their origins. a, EDL differential capacitance near porous graphynes per unit pore surface area. b. Gravimetric EDL capacitance of GDY supercapacitors. $\mathbf{c}$, Number density of ions, $\rho_{\text {ion }}$, inside pore of AA stacking HsGDY under different EDL potential drop. d, Number density of ions, $\rho_{\text {ion }}$, inside pore of AB stacking HsGDY under different EDL potential drop. The upper two lines (in the green zone) are ions in the channel, and the lower two lines (in the red zone) are ions in the cage. e, Radial distribution function, $g(r)$, of cations to anions inside pores of AA and AB stacking HsGDY, and at bulk. f, Interaction energy of ion inside pores of AA and AB stacking HsGDY. $\Delta E$ is the interaction energy difference between ions in the pores and ions in the bulk.

We further scrutinized the EDL capacitance of porous graphyne supercapacitors. The differential EDL capacitance, $C_{\mathrm{D}}$, is calculated by $C_{\mathrm{D}}=\mathrm{d} Q / \mathrm{d} \phi_{\mathrm{D}}$, where $Q$ is surface charge density of 
110

111

112

113

114

115

116

117

118

119

120

121

122

123

124

125

126

127

128

129

130

131

132

133

134

135

136

137

138

139

electrode and $\phi_{\mathrm{D}}$ is the electrostatic potential of the electrode. The AA stacking HsGDY shows a camel shape of the capacitance-potential curve with two maximal values of $8.36 \mathrm{~F} / \mathrm{cm}^{2}$ at $-0.71 \mathrm{~V}$ and $7.95 \mathrm{~F} / \mathrm{cm}^{2}$ at $1.05 \mathrm{~V}$ (Fig. 2a). Such shapes of capacitance-potential curves were also observed for HsGY and HsGTY; however, their specific capacitances are smaller than AA stacking HsGDY (Fig. S6). But for AB stacking HsGDY, the capacitance-potential curve turns into a bell shape with a maximum value of $13.3 \mathrm{~F} / \mathrm{cm}^{2}$ at $0 \mathrm{~V}$. The area-specific EDL capacitance of those porous graphyne supercapacitors is comparable to that of general porous carbon materials. While, porous graphynes could bring excellent gravimetric EDL capacitance and volumetric EDL capacitance (e.g., $220 \mathrm{~F} / \mathrm{g}$ and $130 \mathrm{~F} / \mathrm{cm}^{3}$ for AA stacking HsGDY, $297 \mathrm{~F} / \mathrm{g}$ and $192 \mathrm{~F} / \mathrm{cm}^{3}$ for AB stacking HsGDY) (Fig. 2b and Fig. S7), contributed by their high specific surface area and specific volume.

The capacitance-potential curves could be understood by the potential-dependent ion number density inside pores (Fig. 2c). Under weaker polarization (between $-0.75 \mathrm{~V}$ and $1 \mathrm{~V}$ ), the numbers of cations and anions change faster as polarization is intensified. Under stronger polarization $(<-0.75 \mathrm{~V}$ and $>1 \mathrm{~V})$, the number changes become less pronounced with increasing polarization. Especially when the potential is lower than $-2 \mathrm{~V}$, the anions are almost excluded from the pores, and their number is nearly immune to the potential variation. That accounts for the dual-peak capacitance-potential curve, and smaller EDL capacitance below -2 V. For AB stacking HsGDY, the number density of ions changes the fastest near PZC (Fig. 2d), corresponding to the maximum EDL capacitance obtained at $0 \mathrm{~V}$. Surprisingly, counterions in the channel of AB stacking HsGDY would abnormally decrease because some counterions enter into the cage. The smaller anions in the positive electrode are more likely to enter the cage than the bigger cations in the negative electrode. Therefore, the capacitance of EDL near the positive electrode is higher than that near the negative electrode (Fig. 2a).

The analyses of ion packing inside pores reveal that ions in AA stacking pores gather more closely to each other than those at bulk (Fig. 2e), leading to stronger interactions (Fig. 2f). While ion distribution and interaction energy in $\mathrm{AB}$ stacking pores are closer to those at bulk due to the screening effect of the electrode. Weaker interactions between ions in AB stacking HsGDY make for a lower steric effect, resulting in more convenient ion transport. ${ }^{26}$ That explains why the capacitance of EDL near AB stacking HsGDY is higher than that near AA stacking HsGDY. 


\section{Quantum capacitance}

141 We then investigated the quantum capacitance of porous graphyne supercapacitors. The applied voltage to the device equals the difference of electrochemical potentials between two electrodes, and the electrochemical potential is composed of chemical potential and electrostatic potential ${ }^{27}$.

144 The influence of chemical potential is negligible for well-conductive electrodes (e.g., metal) but is significant for semiconductors (Fig. 3a). For the latter, the electrostatic potential difference equals electrochemical potential difference minus chemical potential difference. As a result, the actual electrostatic potential drop of the EDL is smaller than the applied voltage, and it is necessary to quantify this effect in molecular simulations of supercapacitors with semiconductive electrodes.

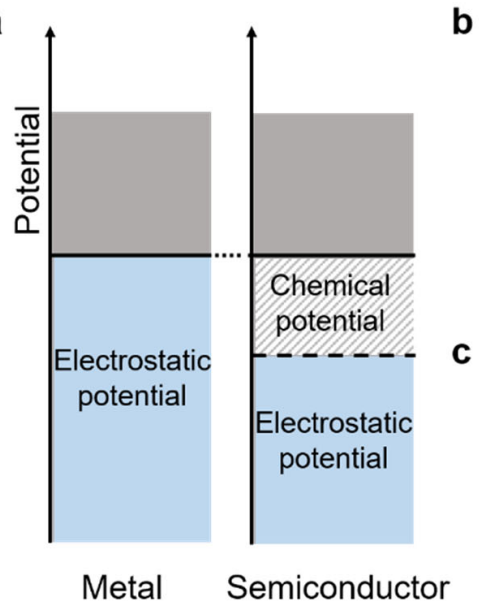

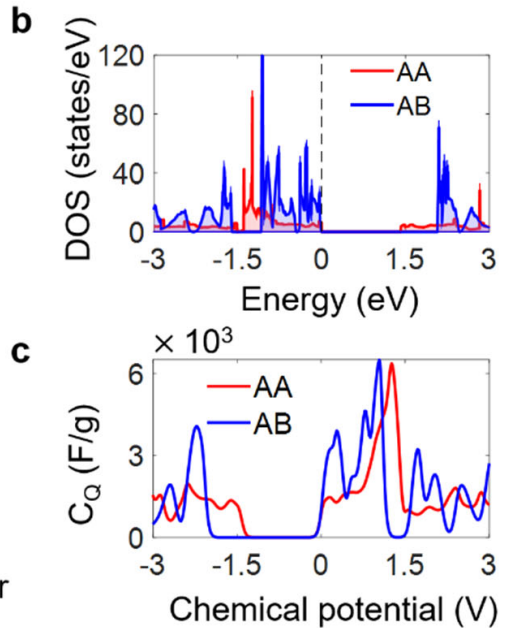

d

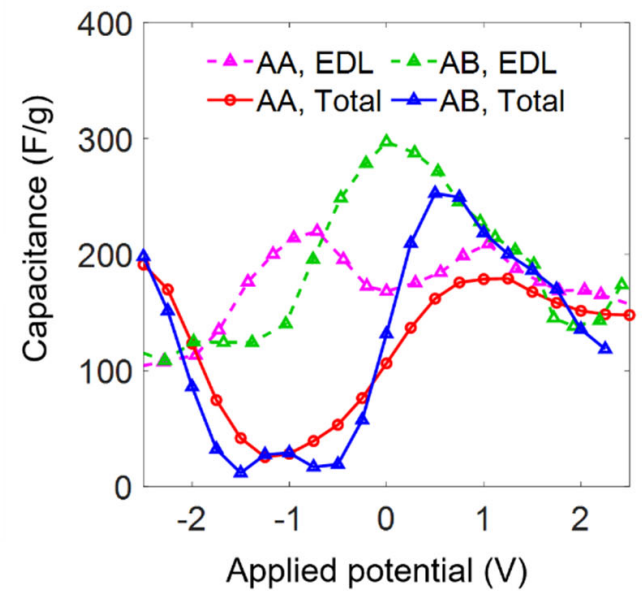

151

152

153

154

155

156

157

158

159

160

161

Fig. 3. Quantum capacitance. a, Schematic of the potential for metal and semiconductor. b-c,Density of state (b) and quantum capacitance (c) of AA (red lines) and AB stacking (blue lines) HsGDY. d, Gravimetric EDL capacitance (dotted lines) and total capacitance (solid lines) of AA and AB stacking HsGDY supercapacitors.

We developed quantum capacitance theory in supercapacitors and clarified the related terminologies (details see Methods and Supplementary part 4). In short, the chemical potential of the electrode is a function of its excess charge, and the proportionality coefficient is quantum capacitance, which can be calculated from the DOS of the electrode. The quantum capacitance is calculated as:

$$
C_{Q}\left(\phi_{\text {chemical }}\right)=\frac{d Q_{\phi}}{d \phi_{\text {chemical }}}=e^{2} \int_{-\infty}^{+\infty} D(E) F_{T}\left(E+e \phi_{\text {chemical }}\right) d E,
$$

where $D(E)$ is the DOS, $F_{T}$ is the thermal broadening function and $\phi_{\text {chemical }}$ is the chemical 
162

163

164

165

potential. $E$ is the relative energy with respect to Fermi level, and $e$ is the elementary charge. Notably, the sign of $e \phi_{\text {chemical }}$ must be positive. Therefore, the total capacitance, $C_{T}$, can be regarded as the sum of quantum capacitance and EDL capacitance in series (Fig. S8):

$$
\frac{1}{C_{T}}=\frac{1}{C_{D}}+\frac{1}{C_{Q}} .
$$

As the DOS of graphynes shown in Fig. 3b, band gaps are observed in both HsGDYs (1.41 $\mathrm{eV}$ for $\mathrm{AA}$ stacking one and $2.07 \mathrm{eV}$ for $\mathrm{AB}$ stacking one), evidencing that they are typical semiconductors with non-negligible quantum capacitance. Similar band gaps are also observed in HsGY (1.72 eV) and HsGTY (1.21 eV) (Fig. S9). Figure 3c depicts the quantum capacitances of both HsGDYs. One can find that, different from the graphene ${ }^{22}$, the quantum capacitance of porous graphynes exhibits obviously asymmetric feature. The quantum capacitances of porous graphynes are quite large under positive polarization, even $6000 \mathrm{~F} / \mathrm{g}$, much higher than that of graphene ${ }^{22}$, and thus have little effect on total capacitances. But under negative polarization, the quantum capacitances are so small due to the band gap that they would dominate the total capacitance.

As predicted, the total capacitances under the positive polarization approximate the EDL capacitances, while those for the negative electrode are lowered quite a lot (e.g., $25 \mathrm{~F} / \mathrm{g}$ at -1.25 V for AA stacking HsGDY, Fig. 3d), which would eventually limit the practical application of porous graphynes as electrodes.

\section{Doping enhances capacitance}

The relatively wide band gaps of HsGDYs will lead to low quantum capacitance and conductivity ${ }^{17}$, and thus reduce the energy and power densities of the device. It has been revealed that the quantum capacitance ${ }^{28,29}$ and conductivity ${ }^{30}$ of graphene could be enhanced by boron (B) or nitrogen $(\mathrm{N})$ doping. We predicted the structures of AA stacking HsGDY with B or N-doped by first-principles calculations to improve their capacitive performance (Fig. S10a). The results of DOS reveal that Fermi levels of all doped HsGDYs shift to the conduction band or valence band (Fig. 4a, Fig. S10b and S11a), meaning that HsGDYs transit from semiconductor to conductor after doping. Specifically, the electronegativity of B (N) is lower (higher) than carbon, leading to electron deficiency (electron injection) and down-shift (up-shift) of the Fermi level. The higher doping concentration of $\mathrm{B}(\mathrm{N})$ leads to more electron deficiency (electron injection), 
resulting in further down-shift (up-shift) of the Fermi level (Fig. 4a and Fig. S11a).

a

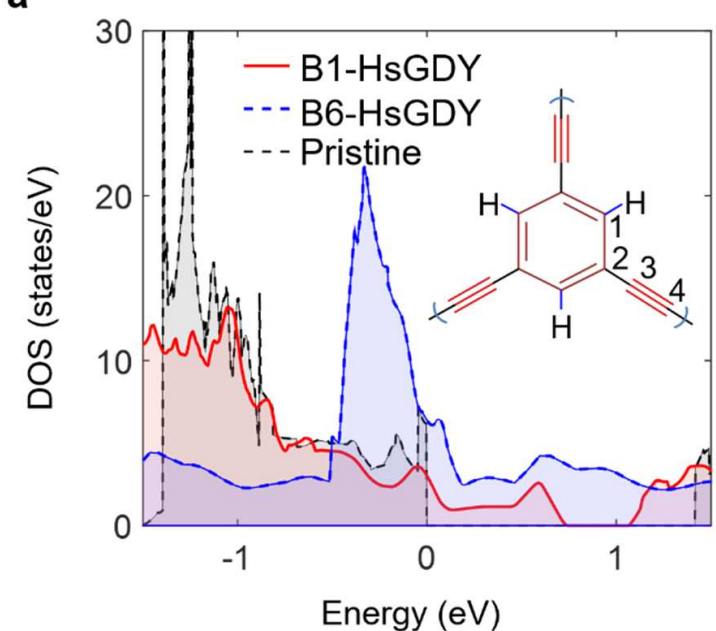

C

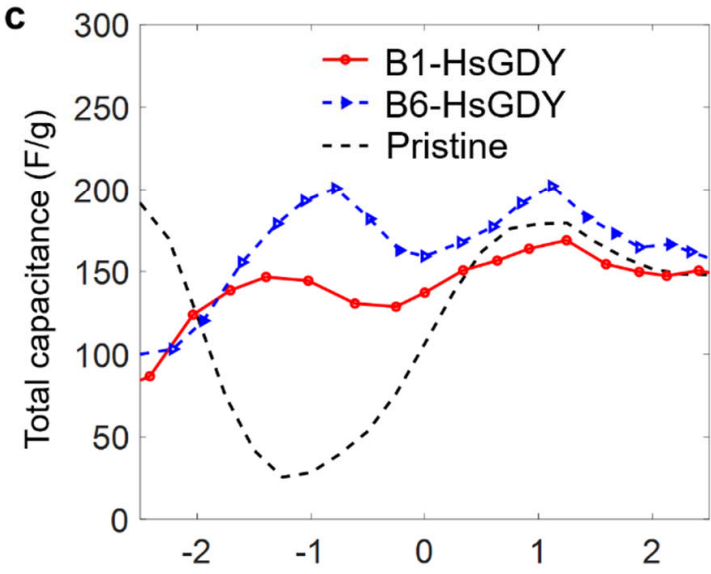

Applied potential (V) b $\times 10^{3}$

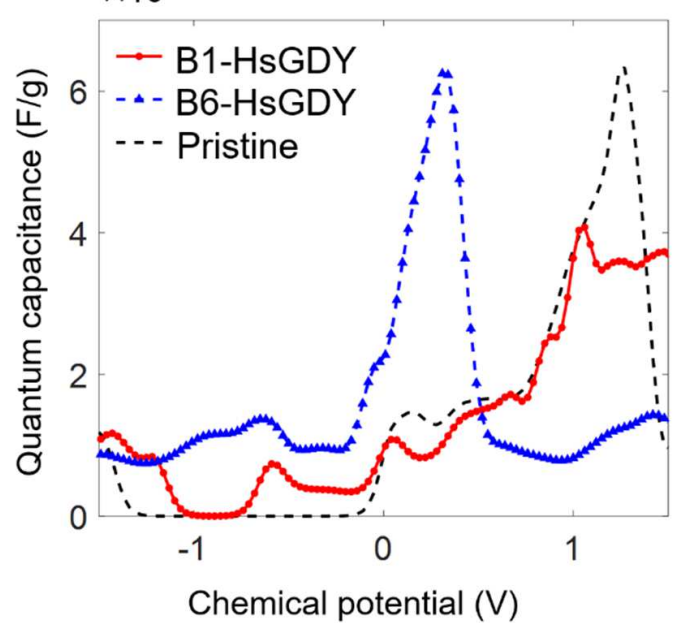

d

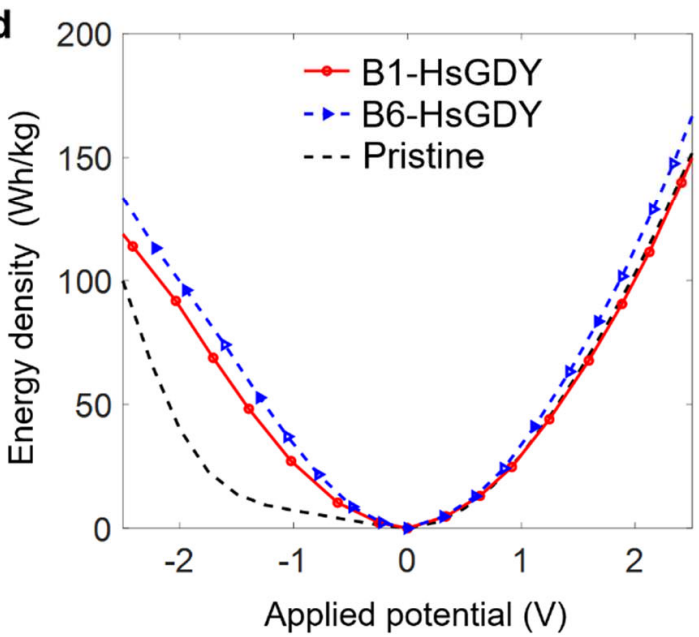

Fig. 4. Doping enhances capacitance performance. a, DOS of B-doped HsGDY. b, Quantum capacitance of B-doped HsGDY. c-d, Gravimetric total capacitance (c) and energy density (d) of B-doped HsGDY. B1-HsGDY (red lines) and B6-HsGDY (blue lines) represent the cases where one or six carbon atoms are doped by boron atoms, respectively, in every two benzene rings (unit cell).

As exhibited in Fig. 4b, Fig S10c and S11b, the quantum capacitance of doped HsGDY is much higher than that of the pristine one and could reach $1000 \mathrm{~F} / \mathrm{g}$ at $0 \mathrm{~V}$ even with lower doping concentrations (i.e., B1-HsGDY and N1-HsGDY). The quantum capacitance of B6HsGDY has a rather high quantum capacitance, reaching $6246 \mathrm{~F} / \mathrm{g}$ at $0.31 \mathrm{~V}$. Therefore, the doping of $\mathrm{B} / \mathrm{N}$ could noticeably improve the quantum capacitance and, thereby, reduce the influence on the total capacitance.

We also examined how doping influences EDL structure and capacitance. It can be noted 
that, despite its high doping concentration, the structure and capacitance of double layers in N6HsGDY supercapacitors are almost the same as those in the pristine one (Fig. S12a-b). A similar result was reported by $\mathrm{MD}$ simulations with graphene and IL [BMIM][PF6] (the EDL capacitance of $\mathrm{N}$-doped graphene supercapacitor is similar to that of pristine graphene) ${ }^{31}$. Therefore, the total capacitance of the doped HsGDY can be simply evaluated from the quantum capacitance of the doped HsGDY (e.g., Fig. 4b) and EDL capacitance of the pristine HsGDY supercapacitor (e.g., Fig. 2b) using Eq. (2). Unlike pristine HsGDY, the total capacitance of doped HsGDYs is dominated by EDL capacitance rather than quantum capacitance (Fig. 4c and Fig. S11c). Consequently, the capacitance-potential curves display double-humped shapes. However, the total capacitance of B1-HsGDY (N1-HsGDY) is still reduced by about $20 \%$ compared with EDL capacitance under negative (positive) polarization. For B6-HsGDY, the influence of quantum capacitance is almost negligible. The total capacitance in the positive and negative electrodes of B6-HsGDY shows good symmetry, like the EDL capacitance, with two excellent maximal values of $200 \mathrm{~F} / \mathrm{g}$ at $-0.78 \mathrm{~V}$ and $202 \mathrm{~F} / \mathrm{g}$ at $1.11 \mathrm{~V}$.

Taking into account the metallicity of electrodes, the energy storage in the supercapacitor consists of two parts: free energy of electrons in electrode and electrostatic energy in EDL, corresponding to quantum capacitance and EDL capacitance ${ }^{32}$. The change in free energy of electrons is subtle for ideal conductors but is non-negligible for electrodes with small quantum capacitance. The free energy density of electrons is also called band-structure energy, $E^{B S}$, which can be obtained by ${ }^{32}$ :

$$
E^{B S}=\int_{-\infty}^{+\infty} D(\epsilon)[f(E+e \phi)-f(E)] E d E=\int_{0}^{\Phi_{e}} \phi_{e} C_{Q} d \phi_{e}
$$

Meanwhile, the EDL energy density, $E_{D}$, is defined as:

$$
E_{D}=\int_{0}^{\Phi_{D}} \phi_{D} C_{D} d \phi_{D}
$$

Therefore, the total energy density, $E_{T}$, is regarded as the sum of the band-structure energy density and EDL energy density:

$$
E_{T}=\Delta E^{B S}+E_{D}
$$

As seen in Fig. 4d, the energy density of the pristine HsGDY is very small under negative polarization. After doping, the energy density is significantly enhanced, even for low doping concentrations. The energy density of B1-HsGDY is $57.93 \mathrm{Wh} / \mathrm{kg}(28.60 \mathrm{Wh} / \mathrm{kg}$ for the pristine 
one) at $3 \mathrm{~V}$, and an outstanding energy density with $98.89 \mathrm{Wh} / \mathrm{kg}$ could be achieved at $3.82 \mathrm{~V}$ of B6-HsGDY.

It is worth noting that we above focused on quaternary doping. In practice, the N-doped has different $\mathrm{N}$ bonding states, including quaternary doping, pyridinic doping, and pyrrole doping. ${ }^{29}$ Pyridinic N-doped HsGDY has been synthesized and showed an excellent catalytic performance $^{33}$. However, as shown in Fig. S13, there is still a large band gap in pyridinic Ndoped HsGDYs, and thereby, the quantum capacitance has not been enhanced. This would account for why the capacitance of pyridinic N-doped HsGDY is not significantly improved compared to the pristine one ${ }^{20}$.

\section{Charging dynamics and capacitive performance}

As HsGDYs have been transformed into good conductors by doping, we took B6-HsGDY as an example and adopted the transmission line model (TLM) to analyze the charging dynamics of doped HsGDY electrodes. Under jump-wise application of constant potential, the time-evolution of net charge of porous electrode depicted by TLM is in the form of Eq. (6) ${ }^{13}$ :

$$
Q(t)=Q_{\infty}\left\{1-\frac{2}{\pi^{2}} \sum_{n=0}^{\infty} \frac{\exp \left[-\pi^{2}\left(n+\frac{1}{2}\right)^{2}\left(\frac{2 l}{L}\right)^{2} \frac{t}{\tau}\right]}{\left(n+\frac{1}{2}\right)^{2}}\right\},
$$

where $Q_{\infty}$ is the charge when the pore is fully charged, $l$ is the pore volume divided by its surface area, $L$ is the full length of the pore ( $l=0.2, L=6.27 \mathrm{~nm}$ for B6-HsGDY), $\tau$ is the intrinsic relaxation time and can be calculated by fitting MD data. The ionic conductivity inside the pore, $\sigma$, is further calculated by:

$$
\sigma=\frac{C \times l}{\tau} .
$$

Therefore, the charging curve obtained from MD simulations can be fitted with TLM to quantify the charging dynamics (Fig. 5a). The intrinsic relaxation time decreases as the voltage increases (Fig. 5b), which can be explained by in-pore ion packing (Fig. 1b). Under lower polarization, the radial distributions of cations and anions overlap as both counterions and co-ions reside near the wall, causing the conflicting motion paths of adsorbed counterions and desorbed co-ions, which blocks ion transport and thus slowing down the charging dynamics (Fig. 5c). Under higher polarization, cations and anions reside in different regions because co-ions will be excluded from the pore center, so that their motion paths are separate, resulting in faster charging (Fig. 5d). 


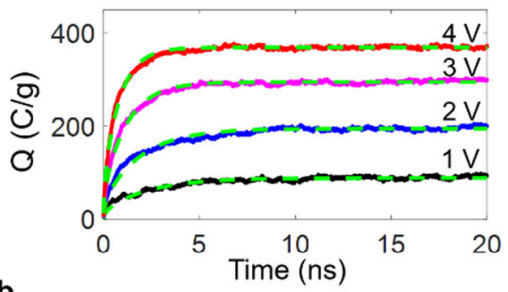

b

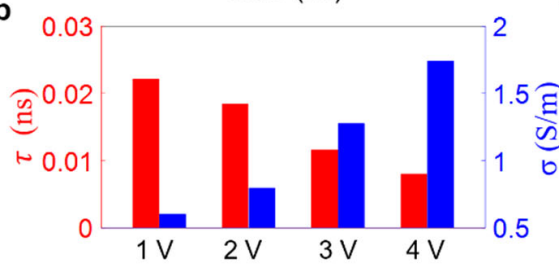

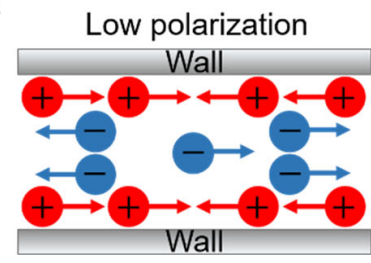

d

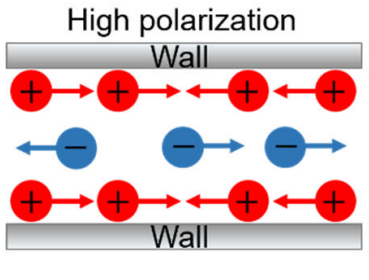

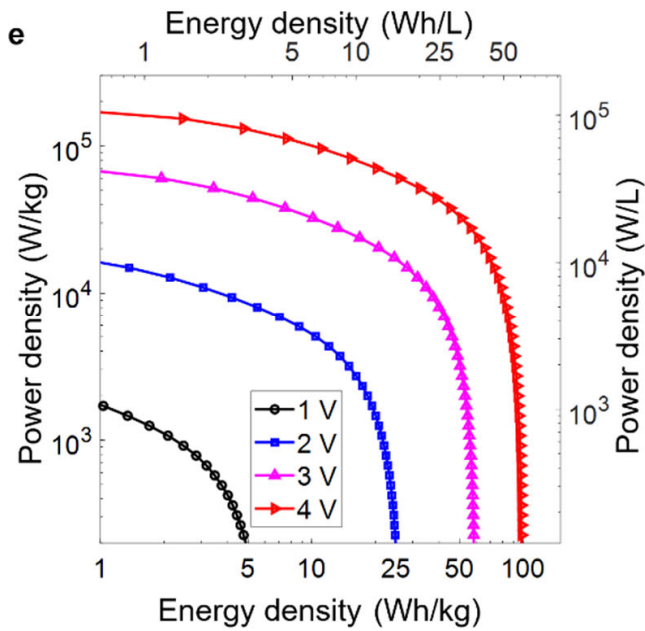

Fig. 5. Charging dynamics and capacitive performance. a, Charging curve, Q, of B6-HsGDY under different voltages. The gravimetric charging curves of B6-HsGDY are obtained by normalized charging curves (e.g., Fig. S12c) versus the specific mass of B6-HsGDY. The colorful solid lines are surface electrode charges obtained by MD simulation, and green dotted lines are the fitted curves by TLM. b, Intrinsic relaxation time $\tau$ (left axis) and the ionic conductivity $\sigma$ (right axis) for B6-HsGDY electrodes with $165 \mu \mathrm{m}$ thickness. c-d, Schematics of motion paths for ions getting into or out of pore at low polarization (c), and high polarization (d). The red and blue spheres represent the cations and anions, respectively. e, Ragone plot of B6-HsGDY supercapacitors under different voltages.

The macroscopic performance of practical crystal electrodes, as made by B6-HsGDY, can be predicted by nanoscale MD data through TLM. We assume a typical size of practical supercapacitor cell consisting of two 165 - $\mu$ m-thick electrodes $\left(L_{c e l l}\right)$ with a diameter $\left(d_{\text {cell }}\right)$ of $16 \mathrm{~mm}$ and a $25-\mu \mathrm{m}$-thick separator (Fig. S14a) based on the previous research ${ }^{34}$. The charging time constant, $\tau_{\text {Cell }}$, of the cell can be roughly estimated as $\tau_{\text {cell }}=\frac{L_{\text {cell }}^{2}}{l^{2}} \tau$. The calculated $\tau_{\text {cell }}$ of B6-HsGDY-based device are 6-16 s (Fig. S14b), much smaller than that of porous carbon (about tens of minutes) ${ }^{35}$. The ionic transport resistance in the electrode can be evaluated by ${ }^{13}$ :

$$
R_{\text {cell }}=\frac{1}{\sigma} \times \frac{L_{\text {cell }}}{\pi\left(\frac{d_{\text {cell }}}{2}\right)^{2} P},
$$

where $P$ denotes the porosity (here is 0.33 ), and the resistances are found to be quite small as $1.47 \sim 4.24 \Omega \mathrm{cm}^{2}$ (Fig. S14b).

The energy and power densities of the device are further calculated based on the capacitance and resistance ${ }^{36,37}$. As shown in the Ragone plot (Fig. 5e), when the applied voltage reaches $3 \mathrm{~V}$, the maximum energy density and power density are $58.71 \mathrm{Wh} / \mathrm{kg}(36.28 \mathrm{Wh} / \mathrm{L})$ and $70.5 \mathrm{~kW} / \mathrm{kg}(43.57 \mathrm{~kW} / \mathrm{L})$, respectively; as the applied voltage reaches $4 \mathrm{~V}$, the maximum energy 

density and power density could reach $98.50 \mathrm{Wh} / \mathrm{kg}(60.86 \mathrm{Wh} / \mathrm{L})$ and $178.85 \mathrm{~kW} / \mathrm{kg}(110.51$ $\mathrm{kW} / \mathrm{L})$ respectively, which surpass most porous carbon supercapacitors. Particularly, the volumetric power density excels all the carbon electrode supercapacitors (Supplementary Table $4)^{2,38-40}$. These power and energy densities are both at the temperature of $400 \mathrm{~K}$. We further calculated the capacitive performance at $300 \mathrm{~K}$. As shown in Fig. S15, the temperature has little effect on the energy density. However, the power density decreases significantly at $300 \mathrm{~K}$ (71.87 $\mathrm{kW} / \mathrm{kg}$ and $44.41 \mathrm{~kW} / \mathrm{L}$ at $4 \mathrm{~V}$ ) but is still higher than most porous carbon supercapacitors.

\section{Discussion}

In this work, we combined MD simulations and DFT calculations as well as the TLM to investigate the charge storage of porous graphynes in IL electrolytes. The constant-potential MD simulations were performed to study the microstructures and interaction energy of IL electrolytes inside different porous graphyne electrodes and their effect on the EDL capacitances. However, the low quantum capacitances of HsGDYs limit their total capacitances. We further predicted the structures and performance of B/N-doped HsGDYs. Results show that doping converts porous graphynes from semiconductors to conductors and thus increases their quantum capacitances on a large scale. The doped porous graphynes, therefore, could achieve high total capacitances and energy density. Finally, the charging dynamics and capacitive performance from nanoscale to macroscale were evaluated by combining MD simulation and TLM. The doped porous graphynes are found to be promising electrodes for supercapacitors with both high energy and power densities.

This work built a multi-scale simulation method from the atomic scale to macroscale for 2D porous electrodes. Although we took porous graphynes as an example, the method may be applied to other 2D porous materials, such as $\mathrm{MOFs}^{41}$ and $\mathrm{COFs}^{42}$.

\section{Methods}

\section{Structure optimization and property calculation of graphynes}

The geometry optimization of each graphyne was derived from DFT calculations as implemented in the Vienna ab-initio simulation package (VASP) ${ }^{43}$ (Supplementary Table 1). The PerdewBurke-Ernzerhof (PBE) ${ }^{44}$ exchange-correlation functions of generalized gradient approximation (GGA) with Grimme's D3 dispersion correction with Becke-Jonson damping ${ }^{45}$ were employed in 
DFT calculations. The projector augmented wave (PAW) method ${ }^{46}$ with a cutoff energy of 550 $\mathrm{eV}$ was used to describe the interaction between nuclei and electrons. The periodic images of atoms were used in all three directions. A k-point mesh of $3 \times 3 \times 11$ and a convergence criterion for the electronic self-consistent loop of $10^{-5} \mathrm{eV}$ were adopted in both structural optimization and DOS calculation. All the atomic positions are fully optimized until all components of the residual forces are smaller than $0.03 \mathrm{eV} \AA^{-1}$.

The structure properties (i.e., pore size, specific surface area, and pore volume) for porous graphynes were calculated by Monte Carlo methods using Zeo++ software ${ }^{47}$ with a nitrogen probe radius of $0.186 \mathrm{~nm}$ (Supplementary Table 2).

\section{MD simulation}

As shown in Fig. S1, each simulation system consists of a pair of porous graphynes as the electrodes (the left one as the negative electrode and the other as the positive electrode), and two RTIL reservoirs separate the pores. Two stacking structures of HsGDY (AA stacking and AB stacking) and porous with different sizes (HsGY and HsGTY) were investigated. The LennardJones model of graphyne atoms was taken from the generic universal force field ${ }^{48}$, and a coarsegrained model of [EMIM][BF 4 was developed by Merlet et al. ${ }^{49}$. All simulations were performed by customized GROMACS software ${ }^{50}$. The electrolyte temperature was maintained at $400 \mathrm{~K}$ using the v-rescale thermostat in the NVT ensemble. The electrostatic interactions were computed in PME method ${ }^{51}$, and the FFT grid spacing was $0.1 \mathrm{~nm}$. A cutoff distance of $1.2 \mathrm{~nm}$ was used in the calculation of electrostatic interactions in the real space.

In our simulations, the applied potential between two porous graphynes electrodes is maintained by the constant potential method (CPM), which allows the fluctuations of changes on electrode atoms during simulation ${ }^{52-54}$. Apparently, this approach is more realistic than the often used constant-charge method, as it considers the electronic polarizability of the electrode and the image force effects ${ }^{55}$. The porous graphynes typically have a 3D rough surface, so that the local redistribution of charges on the electrode due to interaction with electrolyte could not be neglected $^{37,52}$. Besides, the constant charge simulation approach leads to an unphysical enhancement of the charging dynamics ${ }^{56,57}$, so it is essential to use the CPM to study the charging dynamics. The CPM method was implemented in a methodology proposed by Siepmann et $a l^{58}$ and refined by Reed et al. ${ }^{59}$. The system was first held for $20 \mathrm{~ns}$ for 
equilibration without polarization. To obtain EDL structure and capacitance inside the pore, MD simulations were performed under the cell voltage ranging from 0 to $6 \mathrm{~V}$. In this approach, the charge on each electrode was updated at each time step (2 fs).

\section{Calculation of quantum capacitance}

This theory of quantum capacitance is based on fixed-band approximation (i.e., the electronic structure remains unchanged during charging ${ }^{60}$ ). We assume that the electrochemical potential of electrons is the summation of chemical potential and electrostatic potential, and that chemical potential only depends on the number of electrons ${ }^{27}$.

We first discuss the condition at the temperature of $0 \mathrm{~K}$. The numbers of electrons inside the electrode at the neutral state and the polarized state are respectively described as:

$$
\begin{gathered}
n_{0}=\int_{-\infty}^{E_{F}} D(E) d E=\int_{-\infty}^{\mu_{0}} D(E) d E \\
n_{\phi}=\int_{-\infty}^{\bar{\mu}} D(E+e \phi) d E=\int_{-\infty}^{\mu_{\phi^{-}}-e \phi} D(E+e \phi) d E=\int_{-\infty}^{\mu_{\phi}} D(E) d E .
\end{gathered}
$$

where $\mathrm{D}(\mathrm{E})$ is the density of states, $E_{F}$ is the Fermi level, $\mu_{0}$ and $\mu_{\phi}$ are the chemical potential at the neutral state and the polarized state, respectively, $\bar{\mu}$ is the electrochemical potential, and $\phi$ is the electrostatic potential of the electrode.

The net charge of the electrode is

$$
Q_{\phi}=(-e)\left(n_{\phi}-n_{0}\right)=-e \int_{\mu_{0}}^{\mu_{\phi}} D(E) d E
$$

Here we introduce a new concept $\phi_{\text {chemical }}$ called chemical potential in volt as $\delta \mathrm{E}[\mathrm{N}] / \delta \mathrm{Q}$, which means the change in free energy as the change of the charge. The aim is to introduce a physical quantity that has the same dimension as electrostatic potential so that we can use them in an equivalent circuit

$$
\phi_{\text {chemical }}=\frac{\delta E[N]}{\delta Q}=\frac{\delta E[N]}{\delta N} \frac{\delta N}{\delta Q}=-\frac{1}{e} \mu
$$

Analogous to EDL capacitance, the definition of quantum capacitance is defined as

$$
C_{Q}\left(\phi_{\text {chemical }}\right)=\frac{d Q_{\phi}}{d \phi_{\text {chemical }}}=e^{2} D\left(-e \phi_{\text {chemical }}\right) .
$$

The main difference between this quantum capacitance from that of the same name in the field of 
transistors is the negative sign. Only in this way can we set up the equivalent circuit model as quantum capacitance and EDL capacitance in series.

To consider the effects of temperature, the thermal broadening function, $F_{T}(E)=$ $\operatorname{sech}^{2}(E / 2 k T) / 4 k T$, needs to be included as shown in Eq. (1). More information could be found in Supplementary part 4.

\section{Acknowledgements}

The authors in HUST acknowledge the funding support from the National Natural Science Foundation of China (51876072) and the Hubei Provincial Natural Science Foundation of China (2019CFA002, 2020CFA093). They also thank Beijing PARATERA Tech CO., Ltd. for providing HPC resources to accomplish simulations in this work and the support from the Program for HUST Academic Frontier Youth Team.

\section{Author contributions}

G.F. conceived this research. M.T.M. carried out all MD simulations; Z.X.W. did all DFT calculations. T.M.M. and Z.X.W. drafted the paper. G.F., M.T.M., Z.X.W., L.Z., and M.C. analyzed the data. All authors contributed to the discussion and revision of the paper.

\section{Competing interests}

The authors declare no competing interests.

\section{Additional information}

Supplementary information is available for this paper at https: 


\section{Reference}

1. Simon P.; Gogotsi Y.; Dunn B., Where do batteries end and supercapacitors begin?, Science 2014, 343 (6176), 1210-1211.

2. Simon P.; Gogotsi Y., Perspectives for electrochemical capacitors and related devices, Nat. Mater. 2020, 19 (11), 1151-1163.

3. Salanne M.; Rotenberg B.; Naoi K.; Kaneko K.; Taberna P. L.; Grey C. P.; Dunn B.; Simon P., Efficient storage mechanisms for building better supercapacitors, Nat. Energy 2016, 1, 16070.

4. Pomerantseva E.; Bonaccorso F.; Feng X.; Cui Y.; Gogotsi Y., Energy storage: The future enabled by nanomaterials, Science 2019, 366 (6468), eaan8285.

5. Shao H.; Wu Y.-C.; Lin Z.; Taberna P.-L.; Simon P., Nanoporous carbon for electrochemical capacitive energy storage, Chem. Soc. Rev. 2020, 49 (10), 3005-3039.

6. Prehal C.; Koczwara C.; Jackel N.; Schreiber A.; Burian M.; Amenitsch H.; Hartmann M. A.; Presser V.; Paris O., Quantification of ion confinement and desolvation in nanoporous carbon supercapacitors with modelling and in situ x-ray scattering, Nat. Energy 2017, 2 (3), 16215.

7. Chmiola J.; Yushin G.; Gogotsi Y.; Portet C.; Simon P.; Taberna P. L., Anomalous increase in carbon capacitance at pore sizes less than 1 nanometer, Science 2006, 313 (5794), 17601763.

8. Forse A. C.; Griffin J. M.; Merlet C.; Carretero-Gonzalez J.; Raji A. R. O.; Trease N. M.; Grey C. P., Direct observation of ion dynamics in supercapacitor electrodes using in situ diffusion NMR spectroscopy, Nat. Energy 2017, 2 (3), 16216.

9. Bo Z.; Yang J. Y.; Qi H. L.; Yan J. H.; Cen K. F.; Han Z. J., Revealing ion transport in supercapacitors with sub-2 nm two-dimensional graphene channels, Energy Stor. Mater. 2020, 31, 64-71.

10. Sheberla D.; Bachman J. C.; Elias J. S.; Sun C. J.; Shao-Horn Y.; Dinca M., Conductive MOF electrodes for stable supercapacitors with high areal capacitance, Nat. Mater. 2017, 16 (2), 220-224.

11. Li W. H.; Ding K.; Tian H. R.; Yao M. S.; Nath B.; Deng W. H.; Wang Y. B.; Xu G., Conductive metal-organic framework nanowire array electrodes for high-performance solidstate supercapacitors, Adv. Funct. Mater. 2017, 27 (27), 1702067.

12. Feng D. W.; Lei T.; Lukatskaya M. R.; Park J.; Huang Z. H.; Lee M.; Shaw L.; Chen S. C.; Yakovenko A. A.; Kulkarni A.; Xiao J. P.; Fredrickson K.; Tok J. B.; Zou X. D.; Cui Y.; Bao Z. A., Robust and conductive two-dimensional metal-organic frameworks with exceptionally high volumetric and areal capacitance, Nat. Energy 2018, 3 (1), 30-36.

13. Bi S.; Banda H.; Chen M.; Niu L.; Chen M.; Wu T.; Wang J.; Wang R.; Feng J.; Chen T.; Dinca M.; Kornyshev A. A.; Feng G., Molecular understanding of charge storage and charging dynamics in supercapacitors with MOF electrodes and ionic liquid electrolytes, Nat. Mater. 2020, 19 (5), 552-558.

14. Yu M.; Dong R.; Feng X., Two-dimensional carbon-rich conjugated frameworks for 
electrochemical energy applications, J. Am. Chem. Soc. 2020, 142 (30), 12903-12915.

15. Yang Z.; Zhang C. F.; Hou Z. F.; Wang X.; He J. J.; Li X. D.; Song Y. W.; Wang N.; Wang K.; Wang H. L.; Huang C. S., Porous hydrogen substituted graphyne for high capacity and ultra-stable sodium ion storage, J. Mater. Chem. A 2019, 7 (18), 11186-11194.

16. Yang Q.; Guo Y.; Yan B.; Wang C.; Liu Z.; Huang Z.; Wang Y.; Li Y.; Li H.; Song L.; Fan J.; Zhi C., Hydrogen-substituted graphdiyne ion tunnels directing concentration redistribution for commercial-grade dendrite-free zinc anodes, Adv. Mater. 2020, 32 (25), 2001755.

17. He J.; Wang N.; Cui Z.; Du H.; Fu L.; Huang C.; Yang Z.; Shen X.; Yi Y.; Tu Z.; Li Y., Hydrogen substituted graphdiyne as carbon-rich flexible electrode for lithium and sodium ion batteries, Nat. Commun. 2017, 8 (1), 1172.

18. He J. J.; Wang N.; Yang Z.; Shen X. Y.; Wang K.; Huang C. S.; Yi Y. P.; Tu Z. Y.; Li Y. L., Fluoride graphdiyne as a free-standing electrode displaying ultra-stable and extraordinary high li storage performance, Energy Environ. Sci. 2018, 11 (10), 2893-2903.

19. Wang N.; He J.; Tu Z.; Yang Z.; Zhao F.; Li X.; Huang C.; Wang K.; Jiu T.; Yi Y.; Li Y., Synthesis of chlorine-substituted graphdiyne and applications for lithium-ion storage, Angew. Chem. Int. Ed. 2017, 56 (36), 10740-10745.

20. Yue Y.; Xu Y.; Kong F.; Li Q.; Ren S., Bulk-synthesis and supercapacitive energy storage applications of nanoporous triazine-based graphdiyne, Carbon 2020, 167, 202-208.

21. Pan H.; Zhang H.; Wang H.; Li J.; Sun Y.; Lu W.; Wang X., Unusual mechanical and electronic behaviors of bulk layered hydrogen substituted graphdiyne under biaxial strain, Appl. Surf. Sci. 2020, 513, 145694.

22. Xia J.; Chen F.; Li J.; Tao N., Measurement of the quantum capacitance of graphene, Nat. Nanotechnol. 2009, 4 (8), 505-9.

23. Ji H.; Zhao X.; Qiao Z.; Jung J.; Zhu Y.; Lu Y.; Zhang L. L.; MacDonald A. H.; Ruoff R. S., Capacitance of carbon-based electrical double-layer capacitors, Nat. Commun. 2014, 5 (1), 3317.

24. Li J.; Pham P. H. Q.; Zhou W.; Pham T. D.; Burke P. J., Carbon-nanotube-electrolyte interface: Quantum and electric double layer capacitance, ACS Nano 2018, 12 (10), 9763 9774.

25. Li J.; Burke P. J., Measurement of the combined quantum and electrochemical capacitance of a carbon nanotube, Nat. Commun. 2019, 10 (1), 3598.

26. Porada S.; Borchardt L.; Oschatz M.; Bryjak M.; Atchison J. S.; Keesman K. J.; Kaskel S.; Biesheuvel P. M.; Presser V., Direct prediction of the desalination performance of porous carbon electrodes for capacitive deionization, Energy Environ. Sci. 2013, 6 (12), 3700-3712.

27. Brews J. R.; Hwang C. J., Electrochemical potential as the sum of chemical and electrical potentials with a generalization to a wider class of perturbations, J. Chem. Phys. 1971, 54 (8), 3263-3268.

28. Jeong H. M.; Lee J. W.; Shin W. H.; Choi Y. J.; Shin H. J.; Kang J. K.; Choi J. W., Nitrogen-doped graphene for high-performance ultracapacitors and the importance of nitrogen-doped sites at basal planes, Nano Lett. 2011, 11 (6), 2472-2477. 
29. Wang X.; Sun G.; Routh P.; Kim D.-H.; Huang W.; Chen P., Heteroatom-doped graphene materials: Syntheses, properties and applications, Chem. Soc. Rev. 2014, 43 (20), 7067-7098.

30. Zhang W.; Xu C.; Ma C.; Li G.; Wang Y.; Zhang K.; Li F.; Liu C.; Cheng H.-M.; Du Y.; Tang N.; Ren W., Nitrogen-superdoped 3D graphene networks for high-performance supercapacitors, Adv. Mater. 2017, 29 (36), 1701677.

31. Paek E.; Pak A. J.; Kweon K. E.; Hwang G. S., On the origin of the enhanced supercapacitor performance of nitrogen-doped graphene, J. Phys. Chem. C 2013, 117 (11), 5610-5616.

32. Pastewka L.; Järvi T. T.; Mayrhofer L.; Moseler M., Charge-transfer model for carbonaceous electrodes in polar environments, Phys. Rev. B 2011, 83 (16), 165418.

33. Lv Q.; Si W.; He J.; Sun L.; Zhang C.; Wang N.; Yang Z.; Li X.; Wang X.; Deng W.; Long Y.; Huang C.; Li Y., Selectively nitrogen-doped carbon materials as superior metal-free catalysts for oxygen reduction, Nat. Commun. 2018, 9 (1), 3376.

34. Li Z.; Gadipelli S.; Li H.; Howard C. A.; Brett D. J. L.; Shearing P. R.; Guo Z.; Parkin I. $\mathrm{P}$; Li F., Tuning the interlayer spacing of graphene laminate films for efficient pore utilization towards compact capacitive energy storage, Nat. Energy 2020, 5 (2), 160-168.

35. Breitsprecher K.; Janssen M.; Srimuk P.; Mehdi B. L.; Presser V.; Holm C.; Kondrat S., How to speed up ion transport in nanopores, Nat. Commun. 2020, 11 (1), 6085.

36. Christen T.; Carlen M. W., Theory of ragone plots, J. Power Sources 2000, 91 (2), 210-216.

37. Bi S.; Banda H.; Chen M.; Niu L.; Chen M.; Wu T.; Wang J.; Wang R.; Feng J.; Chen T.; Dinca M.; Kornyshev A. A.; Feng G., Molecular understanding of charge storage and charging dynamics in supercapacitors with MOF electrodes and ionic liquid electrolytes, Nat. Mater. 2020, 19 (5), 552-558.

38. Simon P.; Gogotsi Y., Capacitive energy storage in nanostructured carbon-electrolyte systems, Acc. Chem. Res. 2013, 46 (5), 1094-103.

39. Zhong C.; Deng Y.; Hu W.; Qiao J.; Zhang L.; Zhang J., A review of electrolyte materials and compositions for electrochemical supercapacitors, Chem. Soc. Rev. 2015, 44 (21), 7484539 .

40. Dou Q.; Park H. S., Perspective on high-energy carbon-based supercapacitors, Energy Environ. Mater. 2020, 3 (3), 286-305.

41. Sun L.; Campbell M. G.; Dinca M., Electrically conductive porous metal-organic frameworks, Angew. Chem. Int. Ed. 2016, 55 (11), 3566-79.

42. Meng Z.; Stolz R. M.; Mirica K. A., Two-dimensional chemiresistive covalent organic framework with high intrinsic conductivity, J. Am. Chem. Soc. 2019, 141 (30), 11929-11937.

43. Kresse G.; Furthmüller J., Efficiency of ab-initio total energy calculations for metals and semiconductors using a plane-wave basis set, Comput. Mater. Sci. 1996, 6 (1), 15-50.

44. Perdew J. P.; Burke K.; Ernzerhof M., Generalized gradient approximation made simple, Phys. Rev. Lett. 1996, 77 (18), 3865-3868.

45. Grimme S., Semiempirical gga-type density functional constructed with a long-range dispersion correction, J. Comput. Chem. 2006, 27 (15), 1787-1799. 
46. Blöchl P. E., Projector augmented-wave method, Phys. Rev. B 1994, 50 (24), 17953-17979.

47. Willems T. F.; Rycroft C.; Kazi M.; Meza J. C.; Haranczyk M., Algorithms and tools for high-throughput geometry-based analysis of crystalline porous materials, Microporous Mesoporous Mater. 2012, 149 (1), 134-141.

48. Cornell W. D.; Cieplak P.; Bayly C. I.; Gould I. R.; Merz K. M.; Ferguson D. M.; Spellmeyer D. C.; Fox T.; Caldwell J. W.; Kollman P. A., A second generation force field for the simulation of proteins, nucleic acids, and organic molecules, J. Am. Chem. Soc. 1995, 117 (19), 5179-5197.

49. Merlet C.; Salanne M.; Rotenberg B., New coarse-grained models of imidazolium ionic liquids for bulk and interfacial molecular simulations, J. Phys. Chem. C 2012, 116 (14), 7687-7693.

50. Hess B.; Kutzner C.; van der Spoel D.; Lindahl E., Gromacs 4: Algorithms for highly efficient, load-balanced, and scalable molecular simulation, J. Chem. Theory Comput. 2008, 4 (3), 435-447.

51. Gingrich T. R.; Wilson M., On the ewald summation of gaussian charges for the simulation of metallic surfaces, Chem. Phys. Lett. 2010, 500 (1), 178-183.

52. Merlet C.; Rotenberg B.; Madden P. A.; Taberna P. L.; Simon P.; Gogotsi Y.; Salanne M., On the molecular origin of supercapacitance in nanoporous carbon electrodes, Nat. Mater. 2012, 11 (4), 306-10.

53. Vatamanu J.; Vatamanu M.; Bedrov D., Non-faradaic energy storage by room temperature ionic liquids in nanoporous electrodes, ACS Nano 2015, 9 (6), 5999-6017.

54. Merlet C.; Pean C.; Rotenberg B.; Madden P. A.; Daffos B.; Taberna P. L.; Simon P.; Salanne M., Highly confined ions store charge more efficiently in supercapacitors, Nat. Commun. 2013, 4, 2701.

55. Bedrov D.; Piquemal J.-P.; Borodin O.; MacKerell A. D.; Roux B.; Schröder C., Molecular dynamics simulations of ionic liquids and electrolytes using polarizable force fields, Chem. Rev. 2019, 119 (13), 7940-7995.

56. Merlet C.; Péan C.; Rotenberg B.; Madden P. A.; Simon P.; Salanne M., Simulating supercapacitors: Can we model electrodes as constant charge surfaces?, J. Phys. Chem. Lett. 2013, 4 (2), 264-268.

57. Pean C.; Merlet C.; Rotenberg B.; Madden P. A.; Taberna P. L.; Daffos B.; Salanne M.; Simon P., On the dynamics of charging in nanoporous carbon-based supercapacitors, $A C S$ Nano 2014, 8 (2), 1576-83.

58. Siepmann J. I.; Sprik M., Influence of surface topology and electrostatic potential on water/electrode systems, J. Chem. Phys. 1995, 102 (1), 511-524.

59. Reed S. K.; Madden P. A.; Papadopoulos A., Electrochemical charge transfer at a metallic electrode: A simulation study, J. Chem. Phys. 2008, 128 (12), 124701.

60. Zhan C.; Lian C.; Zhang Y.; Thompson M. W.; Xie Y.; Wu J.; Kent P. R. C.; Cummings P. T.; Jiang D. E.; Wesolowski D. J., Computational insights into materials and interfaces for capacitive energy storage, Adv. Sci. 2017, 4 (7), 1700059. 


\section{Supplementary Files}

This is a list of supplementary files associated with this preprint. Click to download.

- GDYILSIv5.1.pdf 